\title{
Hospital incident command system (HICS) performance in Iran; decision making during disasters
}

Ahmadreza Djalali ${ }^{1{ }^{*}}$, Maaret Castren ${ }^{2 \dagger}$, Vahid Hosseinijenab ${ }^{3+}$, Mahmoud Khatib ${ }^{4 \dagger}$, Gunnar Ohlen ${ }^{5+}$ and Lisa Kurland ${ }^{2+}$

\begin{abstract}
Background: Hospitals are cornerstones for health care in a community and must continue to function in the face of a disaster. The Hospital Incident Command System (HICS) is a method by which the hospital operates when an emergency is declared. Hospitals are often ill equipped to evaluate the strengths and vulnerabilities of their own management systems before the occurrence of an actual disaster. The main objective of this study was to measure the decision making performance according to HICS job actions sheets using tabletop exercises.

Methods: This observational study was conducted between May 1st 2008 and August 31st 2009. Twenty three Iranian hospitals were included. A tabletop exercise was developed for each hospital which in turn was based on the highest probable risk. The job action sheets of the HICS were used as measurements of performance. Each indicator was considered as 1, 2 or 3 in accordance with the HICS. Fair performance was determined as < 40\%; intermediate as 41-70\%; high as $71-100 \%$ of the maximum score of 192. Descriptive statistics, T-test, and Univariate Analysis of Variance were used.
\end{abstract}

Results: None of the participating hospitals had a hospital disaster management plan. The performance according to HICS was intermediate for $83 \%(n=19)$ of the participating hospitals. No hospital had a high level of performance. The performance level for the individual sections was intermediate or fair, except for the logistic and finance sections which demonstrated a higher level of performance. The public hospitals had overall higher performances than university hospitals $(P=0.04)$.

Conclusions: The decision making performance in the Iranian hospitals, as measured during table top exercises and using the indicators proposed by HICS was intermediate to poor. In addition, this study demonstrates that the HICS job action sheets can be used as a template for measuring the hospital response. Simulations can be used to assess preparedness, but the correlation with outcome remains to be studied.

Keywords: Hospital Incident Command System, Performance, Exercise

\section{Background}

Disasters, both natural and man-made, and the number of people affected by them have increased over the past decades [1,2]. The impact is illustrated by 46 million people being affected by earthquakes and tsunamis, between 1991 and 2005, worldwide [2]. Hospitals are

\footnotetext{
* Correspondence: ahmadreza.djalali@ki.se

† Contributed equally

'Karolinska Institutet, Department of Clinical Science and Education,

Södersjukhuset, Stockholm, Sweden

Full list of author information is available at the end of the article
}

cornerstones for health care in a community, and must continue to function in the face of a disaster [3,4]. An effective hospital command system is therefore crucial.

The Incident Command System (ICS) is a standardized on-scene, all-hazards incident management approach designed specifically to allow responders to adopt an integrated organizational structure equal to the complexity and demands of any single incident or multiple incidents without being hindered by jurisdictional boundaries [5,6]. The ICS was created in 1970 in response to a series of wildfires in Southern California

\section{Biomed Central}


in the United States $[7,8]$. The goal was to simplify communication and establish lines to authority and command $[7,8]$, also, to provide more effective onsite utilization and management of resources [9]. The Incident Command System typically consists of five system functions as follows: Command, Planning, Operations, Logistics, and Financial/Administration [5,6].

The ICS was adapted in 1991 for use in the hospital based response to disasters, and duly renamed to the Hospital Emergency Incident Command System (HEICS) [10,11]. HEICS was revised in both 1992 and 1998. The most recent version, namely the Hospital Incident Command System (HICS), was presented in 2006 [12]. The HICS is currently the most commonly used model for hospital disaster response in the United States, also has been used in Taiwan and Turkey [13-18].

HEICS/HICS is a method by which the hospital operates when an emergency is declared [19]. It is the hospital's "standard operating procedure" whenever the hospital's disaster plan is activated, allowing the hospital to activate only those elements needed to address a specific emergency [19]. It also, using a common organizational terminology, facilitates communication between the hospital, first responders and other health-care facilities [19]. The overall objective is to facilitate the hospital response to disasters by superimposing a managerial structure for command and control, in addition to, a coordination of organizational missions e.g. administrative, logistical, informational, financial, and operational tasks [20].

The lack of internationally accepted standards of performance makes it difficult to evaluate the outcome of health care in a disaster [21]. Also, no generally accepted methodology exists for the evaluation of HICS or hospital-based exercises [21]. The evaluation of performance in disaster management is a topic of great interest [22,23]. Performance is a measure of how well an activity is done and can be measured in terms of accuracy, time, and quality $[24,25]$. The managerial activity for each position during the disaster response was determined in the relevant HICS job action sheets [12].

The vulnerability of Iran, with respect to natural disasters, especially earthquakes [26-28], necessitates an effective medical response to disasters. Iran's Ministry of Health and Medical Education required that all hospitals implemented the Hospital Incident Command System in 2007. Basic training courses in disaster medicine and the usage of HICS were developed by the medical science universities to support these efforts and were based on national guidelines [29].

The infrequent occurrence of major disasters is one reason that health care organizations remain ill equipped to systematically evaluate the strengths and vulnerabilities of their emergency management systems and programs [30]. Simulations are, therefore, important tools for the evaluation of disaster management and institutional performance [21,22,31-36]. A tabletop exercise is one type of simulation. It allows staff and key decision makers to discuss and act out an incident response under simulated emergency settings [3].

There has not previously, to our knowledge, been any previous study regarding the impact of the implementation of HICS on hospital performance with respect to response to a real or simulated disaster. The main objective of this study was to measure the decision making performance according to HICS job actions sheets using tabletop exercises.

\section{Methods \\ Setting}

This observational study was conducted in Iran, between May 1st 2008 and August 31st 2009. Hospitals that had implemented a hospital response system in accordance with the HICS and had a basic training course adapted to the HICS and were able to conduct a tabletop exercise were included. Twenty three hospitals were included in this study, due to financial constraints. None of these was excluded. Fourteen of the 23 hospitals were public and 9 were university hospitals. In Iran, there are three types of hospitals; university hospitals, private hospitals, and non-governmental (public) hospitals that belong to different organizations e.g. social security organization

Affiliation (public or university hospital), size (small: less than 100 beds; medium: 100-400 beds; large: more than 400 beds), use of a HICS advanced training course, the presence of a hospital command centre, and a hospital disaster plan, were assessed for each participating hospital.

\section{Table top exercises}

A tabletop exercise was developed for each hospital. Tabletop exercises are simulated situations. Participants discuss the problems at hand, in depth, and make decisions regarding emergency responses, accordingly. One of the most important goals of a tabletop exercise is to compel the participant to make problem solving decisions. The decisions are documented to serve as a reference with which to evaluate the exercise $[3,36,37]$.

Each exercise was based on a risk map of the area. The most likely hazard for each given hospital was chosen as the disaster scenario for the exercise.

Each scenario was run for a maximum of 2 hours. There are 28 standardized scenarios in the HICS-2006 including earthquake, fire and chemical emergencies. The content of these scenarios was extended with 
respect to geographical information, characteristics of the disaster, and process of influx of disaster casualties. A facilitator, one of the evaluators, initiated the discussion and directed the participants toward indepth problem solving. All positions of the HICS were assessed according to the 5 main sections (command, operations, planning, logistics, and finance/administration) using the job action sheets, during both the exercise and the evaluation process [8]. The participants were asked to document all decisions made during the exercise. The number of participants differed due to the size of the participating hospitals. There was one person for each position at 4 hospitals, but in 19 hospitals some personnel was responsible for two positions, e.g. a person for "Medical Gases" and "Medical Devices".

The scoring according to the job action sheets was done by a group of three evaluators. The evaluators were medical doctors, who had worked in the field of HICS and HDP, for at least 6 years. They had international experiences from training programs and visits of hospital using HICS. The same group of evaluators was used to for all 23 exercises. There was a team discussion after each exercise. The about scoring of HICS performance by the evaluators was completed in consensus.

\section{Measurement of performance}

The HICS job action sheets were used as the performance indicators for decision making (see additional file 1) [8]. The job action sheet is an incident management tool designed to familiarize the user with critical aspects of the command position he or she is assuming. It includes title, purpose, to whom they report, and critical action considerations. These tasks are intended to prompt the incident management team members to take needed actions related to their roles and responsibilities [12]. The performance for each HICS position was scored based on the compatibility of the participants' decisions with the relevant job action sheet. A compatibility below $40 \%$ was scored as 1 , a compatibility between 40 and $70 \%$ as 2 , and if the compatibility was above $70 \%$, the score was 3 . The score was 0 if the position was missed or no performance was achieved for the specific task.

The range for the total HICS score was 1-192 (see additional file 1), and was divided into at three categories: Fair: 1-76; Intermediate: 77-134; High: 135-192. These cut-off values are based on expert consensus.

The evaluators compared the participants' documented decisions with the content according to the job action sheets, as a measure of compatibility. The evaluators agreed on to what extent the duties in a job action sheet are provided by the documented decisions of the participant (HICS member).

\section{Statistical analysis}

Descriptive statistics was performed. Measures of central tendency were used for HICS and its main sections performance scores. The HICS scores were normally distributed except for the scores for the planning section, as tested by a normal probability plot [38]. A t-test was used to compare means of HICS performance between hospitals with respect to their affiliation, size and training courses. A Univariate Analysis of Variance was used to evaluate the effect of independent variables on HICS performance. A p-value of less than 0.05 , two tailed, was considered to be significant.

The SPSS 17 (IBM, New York, USA) was used for data analysis.

\section{Results}

The public hospitals had implemented the HICS earlier than the university (community) hospitals; and had also been provided with a budget with which to conduct relevant training programs.

Background information for the participating hospitals is presented in Table 1. The hospitals were located in 12 different cities including Tehran and 1-2 hospitals from each disaster regional collaborating center of Iran's Ministry of Health. Their location is not disclosed in detail due to confidentiality reasons, in accordance with WHO's recommendations. At the time of this study, there were 9 regional disaster centers of Iran's Ministry of Health. None of the participating hospitals had a hospital disaster plan. Only two hospitals had a designated Hospital Command Centre. The exercise included one of the following hazards; earthquake $(n=15)$, accident with hazardous materials $(n=5)$, and fire $(n=3)$.

The lowest total HICS score was 56 and the highest was 119 , with a mean of 85 ( $\pm 15 \mathrm{SD}$ ). The logistic and financial/administration sections received the highest scores, and the planning section had the lowest score (Table 2).

HICS performance was intermediate for 19 hospitals (83\%). No hospital had a high level of performance. The performance level of the different individual sections

\section{Table 1 Background for the $\mathbf{2 3}$ hospitals that participated in the tabletop exercises and HICS performance}

\begin{tabular}{ll}
\hline History of advanced HICS training course* $\mathbf{n}(\%)$ & No $8(35 \%)$ \\
& Yes 15 (65\%) \\
\hline Hospital Disaster Plan $\mathbf{n}(\%)$ & Present $0(0 \%)$ \\
\hline Hospital Command Centre $\mathbf{n}(\%)$ & No $21(91 \%)$ \\
& Yes 2 (9\%) \\
\hline Hospital size** $\mathbf{n}(\%)$ & Small 6 (26\%) \\
& Medium 17 (74\%) \\
\hline
\end{tabular}

*HICS training modules

**Small: less than 100 beds, Medium:100-400 beds 
Table 2 HICS score presented for each section for the 23 participating hospitals.

\begin{tabular}{ccc}
\hline Sections of HICS & Mean \pm SD & Range \\
\hline Command & $5.2 \pm 1.7$ & $3-9$ \\
\hline Operations & $43.1 \pm 7.8$ & $26-58$ \\
\hline Planning & $8.6 \pm 2.9$ & $5-17$ \\
\hline Logistics & $19.1 \pm 3.7$ & $12-26$ \\
\hline Financial/Administration & $9.0 \pm 1.8$ & $6-13$ \\
\hline Total HICS & $85.0 \pm 15.6$ & $56-119$ \\
\hline
\end{tabular}

was fair to intermediate, except for the logistics and finance/administration sections which had a high level of performance in 3 and 5 hospitals, respectively (Figure 1).

The HICS and its main sections in the public hospitals had a higher performance than university hospitals (Table 3), except for the operations section where there was no significant difference in performance $(P=0.07)$.

Hospitals with both basic and advanced courses had a higher level of performance $(89.2 \pm 15.5)$ as compared to hospitals with only the basic course $(77.1 \pm 13.3)$, although the difference was not significant $(\mathrm{P}=0.07)$.

There was no significant difference between small and medium hospitals $(\mathrm{P}=0.09)$. No large hospitals participated in the study.

Public affiliation was the only independent predictor of the HICS performance $(P=0.04)$ in a regression model including medium size, public affiliation, advanced course, and earthquake scenario.

\section{Discussion}

This study showed that the decision making performance, as measured by the indicators proposed by the Hospital Incident Command System (HICS), during tabletop exercises was intermediate for the majority of the participating hospitals and poor in a few, while none showed a high performance. Previous studies evaluating hospital management during simulated disasters demonstrated a similar level of performance [23,39-41]. However, these studies [23,39-41] have only evaluated decision making with respect to the command and control functions, and not the overall organisation. It is necessary to evaluate all components of the hospital disaster response in order to assess the efficacy of the hospital response to a disaster. We believe that one way to accomplish this is by using the HICS job action sheets as a template for measuring performance.

The performance at public hospitals was significantly higher than that of university hospitals. There are, to our knowledge, no previous studies on performance as measured by HICS and hospital affiliation. However public hospitals did not appear to be superior to other hospitals, with respect to disaster preparedness as measured by practice variation, plan characteristics, and surge capacity in a previous study $[42,43]$.

HICS was implemented at public hospitals approximately one year before that of university hospitals. In addition, there is more financial support for training courses and drills at public hospitals as compared to university hospitals. Thus, public hospitals have a longer experience of HICS and are also better funded, which may explain our results. Previous studies demonstrated that funding, standards, and experience in disaster management are the improving factors for hospital disaster preparedness [4].

Training courses had no significant impact on HICS performance according to the current study. Also a review from 2004 showed no support for training courses influencing performance [44]. More recent studies have, however, shown that disaster management training courses enhance individual knowledge and skills [29,45]. The content, construction and execution of the training courses were beyond the scope of this study, which may, in part, influence our results. Interventional studies including standardized training courses are needed.

The hospital size did not affect the performance as measured by HICS according to our results. There are, however, previous studies suggesting that size is an important function of hospital capacity in disaster response [46-48]. However, there is no consensus on size in relation to preparedness [43]. There are no previous reports regarding hospital size and managerial performance as measured by HICS. We believe that hospital performance is an effect of preparedness and not size per se.

HICS was implemented in Iran in 2007. Therefore, one may infer that the lack of high performance may be explained by an insufficient understanding of HICS and also an incompatibility of HICS with the pre-existing management structure. However, we believe that the intermediate to low performance is a consequence of the lack of a comprehensive hospital disaster plan and a hospital command centre (HCC). There are, however, to date, no studies on the effect of implementing a disaster plan on HICS performance.

\section{Limitations}

A limitation of this study is that it is performed in Iran which restricts the generalizability of our results. However, the HICS and its job action sheets are standardized and used worldwide. Despite the lack of internationally accepted standards of performance for disaster health management $[21,30]$ the HICS does have international recognition.

An additional limitation is that we have not measured inter-rater reliability. We did, however, have a team discussion after each exercise and the evaluators 


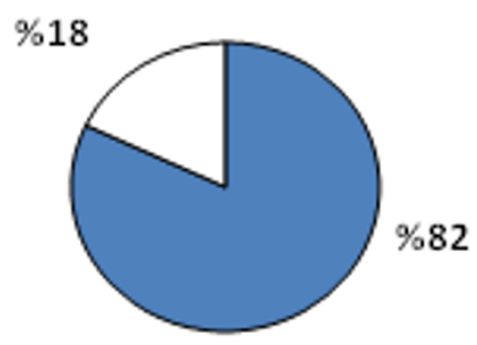

a. HICS

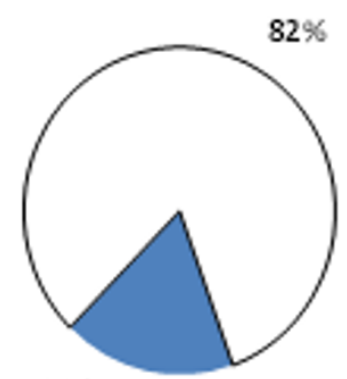

$18 \%$

c. Operations Section

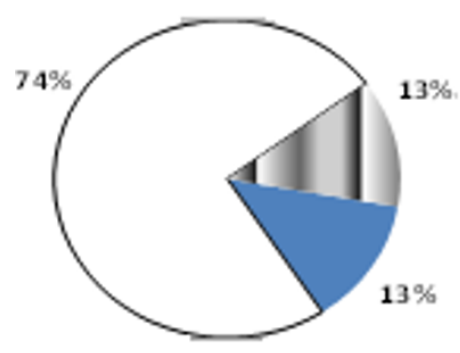

e. Logistics Section

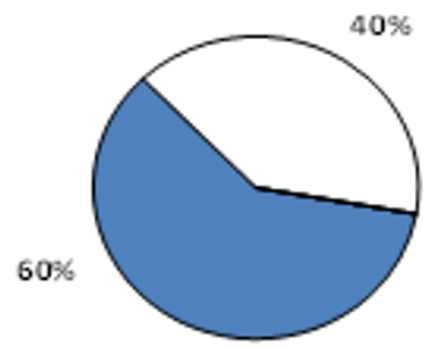

b. Command Section

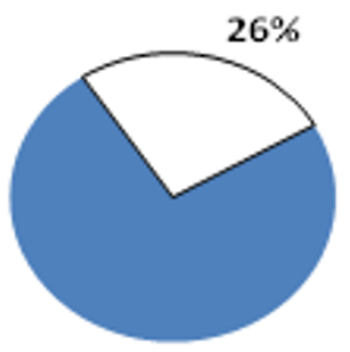

$74 \%$

\section{d. Planning Section}

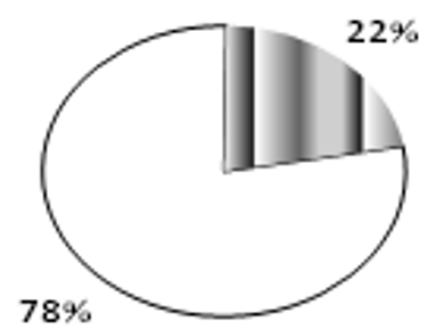

f. Finance/Administration Section

Fair $\square$ Intermediate in High

Figure 1 Performance level as presented in pie charts for a. the total HICS score (a sum of b-f), b. Command Section score, c. Operations Section score, d. Planning Section score, e. Logistics Section score, f. Finance/Administration Section score.

independently came to the same HICS score. Another limitation is that the cut-off levels for the performance levels are arbitrary. However, they were based on expert consensus. Standardizing these cut-off levels requires prospective outcome based studies.

Additionally, there were too few participating hospitals to allow for the testing the impact of different scenarios for the same hospital. Only one scenario was used for each hospital, which limits the results to these hazards.
Other hazards would be of interest, such as hazardous materials, fire and power shortage. However, the hazard chosen for each hospital was that of highest risk.

Using tabletop exercises may of course be questioned with respect to the validity of assessing hospital preparedness [31-36]. However, decision making performance, as measured in tabletop exercises, has impact on preparedness as measured in other functional exercises [41]. Outcome studies remain to be performed. 
Table 3 Results of the tabletop exercise presented as the HICS score for each section for the 23 participating hospitals in relation to their affiliation, ie.

\begin{tabular}{cccc}
\hline Sections of HICS & Mean \pm SD & $\begin{array}{l}\text { p-value } \\
(\mathbf{2}- \\
\text { tailed) }\end{array}$ \\
\hline & $\begin{array}{c}\text { University }(\mathbf{n}= \\
\text { 9) }\end{array}$ & $\begin{array}{c}\text { Public }(\mathbf{n}= \\
\mathbf{1 4})\end{array}$ & \\
\hline Command & $4.1 \pm 0.8$ & $5.9 \pm 1.7$ & 0.008 \\
\hline Operations & $39.4 \pm 8.8$ & $45.5 \pm 6.4$ & 0.07 \\
\hline Planning & $7.1 \pm 0.8$ & $9.5 \pm 3.2$ & 0.04 \\
\hline Logistics & $16.7 \pm 2.3$ & $20.6 \pm 3.7$ & 0.009 \\
\hline Financial/ & $7.7 \pm 0.7$ & $9.9 \pm 1.8$ & 0.001 \\
\hline Administration & & & \\
\hline Total HICS & $75.0 \pm 11.9$ & $91.4 \pm 14.5$ & 0.01 \\
\hline
\end{tabular}

whether they are public or university hospitals.

\section{Conclusions}

The decision making performance in the participating Iranian hospitals, as measured during tabletop exercises and using the Hospital Incident Command System (HICS), was intermediate to poor. The performance was better in the public hospitals as compared to university hospitals and was shown to be independent of the hospital size.

The HICS job action sheets can be used as a template for measuring the hospital response. We believe that a comprehensive hospital disaster plan should include not only managerial and operational elements of hospital preparedness, but also an appropriate command system suited to the specific hospital organisation.

Simulations can be used to assess preparedness, but the correlation with outcome remains to be studied.

\section{Additional material}

Additional file 1: Indicators of decision making performance of Hospital Incident Command System in five different sections; achieved results on each indicator given $0,1,2$, or 3 points.

List of Abbreviations

HICS: Hospital Incident Command System

\section{Author details}

${ }^{1}$ Karolinska Institutet, Department of Clinical Science and Education, Södersjukhuset, Stockholm, Sweden. ${ }^{2}$ Karolinska Institutet, Department of Clinical Sciences and Education and Department of Emergency Medicine, Södersjukhuset, Stockholm, Sweden. ${ }^{3}$ Department of emergency management, Natural Disaster Research Institute, Tehran, Iran. ${ }^{4}$ Tehran social security organization, Tehran, Iran. ${ }^{5}$ Karolinska Institutet, Department of Clinical Science, Intervention and Technology, Stockholm, Sweden.

\section{Authors' contributions}

ARD was involved in the study design, data collection, analysis, and manuscript writing. $\mathrm{MC}$ contributed to the analysis of the data and to the writing of the manuscript. VH and MK were involved in the study design and took an active part in the data collection and the interpretation of the results. GO participated in the study design, writing-up and finalization of the manuscript. LK participated in the study design, analysis and the manuscript writing, revision and editing. All authors read and approved the final manuscript.

\section{Competing interests}

The authors declare that they have no competing interests.

Received: 21 November 2011 Accepted: 6 February 2012

Published: 6 February 2012

\section{References}

1. Disaster Trends, World 1900-2010. EM-DAT, International Disaster Database , [http://www.emdat.be]. Accessed June 2011.

2. Disaster statistics 1991-2005. International Strategy for Disaster Reduction, [http://www.unisdr.org/disaster-statistics/occurrence-trends-century.htm]. Accessed December 2010

3. Hospital Preparedness Exercises Guidebook. Agency for Healthcare Research and Quality, [www.ahrq.gov/prep/hospexguide/hospexguide.pdf]. Accessed May 2011.

4. Barbera JA, Yeatts DJ, Macintyre AG: Challenge of hospital emergency preparedness: analysis and recommendations. Disaster Med Public Health Prep 2009, 3(2 Suppl):S74-82.

5. Incident Command System (ICS) Overview. Federal Emergency Management Agency, [www.fema.gov/emergency/nims/ IncidentCommandSystem.shtm\#item3]. Accessed December 2012.

6. Incident Command System (ICS). Occupational Safety and Health Administration, [www.osha.gov/SLTC/etools/ics/what_is_ics.html]. Accessed December 2012

7. Londorf D: Hospital application of incident management system. Prehospital and Disaster Medicine 1995, 10(3):184-188.

8. Bailin MT, et al: Incident command system. In Advanced Disaster Medical Response.. 1 edition. Edited by: Briggs SM, Brinsfield KH. Boston, MA: Harvard Medical International Trauma 2003:3-5

9. Chase RA: FIRESCOPE: A new concept in multi-agency fire suppression coordination. Berkeley, CA: United States Department of Agriculture, Forest Service, Pacific Southwest Forest and Range Experiment Station; 1980, General Technical Report PSW-40;

10. Zane RD, Prestipino AL: Implementing the Hospital Emergency Incident Command System: An integrated delivery system's experience. Prehosp Disast Med 2004, 19(4):311-317.

11. Hospital Emergency Incident Command System, Version 3, 1998. San Mateo County Health Services Agency Emergency Medical Services, [http:// www.heics.com/HEICS98a.pdf]. Accessed April 2011.

12. Hospital Incident Command System Guidebook, 2006. The California Emergency Medical Services Authority (EMSA), [http://www.emsa.ca.gov/hics/ ]. Accessed April 2011.

13. Arnold JL, Dembry L, Tsai MC, et al: Recommended modifications and applications of the Hospital Emergency Incident Command System for hospital emergency management. Prehosp Disast Med 2005, 20(5):290-300

14. Wang TL, Chang H: Appraisal of Disaster Response Plan of Hospitals in Taipei Judged by Hospital Emergency Incident Command System (HEICS). Ann Disaster Med 2003, 1:104-111.

15. Wang $\mathrm{TL}$, Chang H: Application of Evidence-Based Hospital Emergency Incident Command System (HEICS) in Taipei. Ann Disaster Med 2006, 4(2):54-59.

16. Tsai MC, Arnold JL, Chuang CC, et al: Implementation of the Hospital Emergency Incident Command System during an outbreak of severe acute respiratory syndrome (SARS) at a hospital in Taiwan, ROC. J Emerg Med 2005, 28(2):185-96.

17. Autrey P, Moss J: High-reliability teams and situation awareness: implementing a hospital emergency incident command system. J Nurs Adm 2006, 36(2):67-72

18. Arnold J, O'Brien D, Walsh D, et al: The perceived usefulness of the Hospital Emergency Incident Command System and an assessment tool for hospital disaster response capabilities and needs in hospital disaster planning in Turkey (abstract). Prehosp Disast Med 2001, 16(2):s12.

19. Born CT, Briggs SM, Ciraulo DL, et al: Disasters and mass casualties: I. General principles of response and management. J Am Acad Orthop Surg 2007, 15(7):388-96 
20. Arnold JL, Paturas J, Rodoplu U: Measures of effectiveness of hospital incident command system performance. Prehosp Disaster Med 2005, 20(3):202-5

21. Thomas TL, Hsu EB, Kim HK, et al: The incident command system in disasters: Evaluation methods for a hospital-based exercise. Prehosp Disast Med 2005, 20(1):14-23.

22. Nilsson $H$, Vikström $T$, Rüter A: Quality control in disaster medicine training-initial regional medical command and control as an example. Am J Disaster Med 2010, 5(1):35-40.

23. Rüter A, Nilsson H, Vilkström T: Performance indicators as quality control for testing and evaluating hospital management groups: A pilot study. Prehosp Disast Med 2006, 21(6):423-426.

24. Cambridge dictionary., [http://dictionary.cambridge.org]. Accessed October 2011.

25. Public health emergency exercise toolkit. Columbia University, [http:// www.nursing.columbia.edu/pdf/PublicHealthBooklet_060803.pdf]. Accessed May 2011.

26. Djalali A, Khankeh $H$, Öhlén $G$, et al: Facilitators and obstacles in prehospital medical response to earthquakes: a qualitative study. Scand J Trauma Resusc Emerg Med 2011, 16(19):30.

27. National report of the Islamic Republic of Iran on disaster reduction. World Conference on Disaster Reduction 2005, Kobe, Hyogo, Japan. [http:// www.unisdr.org/eng/mdgs-drr/national-reports//ran-report.pdf]. Accessed June 2011

28. A global report: Reducing disaster risk a challenge for development. United Nations Development Programme 2004, [http://www.undp.org/cpr/ disred/documents/publications/rdr/english/rdr_english.pdf]. Accessed January 2011.

29. Djalali A, Hosseinijenab V, Hasani A, et al: A fundamental, national, disaster management plan: An education based model. Prehosp Disaster Med 2009, 24(6):565-569.

30. Lazar EJ, Cagliuso NV Sr, Gebbie KM: Are we ready and how do we know? The urgent need for performance metrics in hospital emergency management. Disaster Med Public Health Prep 2009, 3(1):57-60.

31. Savoia E, Testa MA, Biddinger PD, et al: Assessing public health capabilities during emergency preparedness tabletop exercises: reliability and validity of a measurement tool. Public Health Rep 2009, 124(1):138-48.

32. Biddinger PD, Savoia E, Massin-Short SB: Public health emergency preparedness exercises: lessons learned. Public Health Rep 2010, 125(Suppl 5):100-6.

33. Franc-Law JM, Bullard M, Della Corte F: Simulation of a hospital disaster plan: A virtual, live exercise. Prehosp Disast Med 2008, 23(4):346-353.

34. Bartley BH, Stella JB, Walsh LD: What a disaster?! Assessing utility of simulated disaster exercise and associated educational process. Prehosp Disast Med 2006, 21(4):249-255.

35. Dausey DJ, Buehler JW, Lurie N: Designing and conducting tabletop exercises to assess public health preparedness for manmade and naturally occurring biological threats. BMC Public Health 2007, 29(7):92

36. Gebbie KM, Valas J, Merrill J, et al: Role of exercises and drills in the evaluation of public health in emergency response. Prehosp Disast Med 2006, 21(3):173-182.

37. Bradshaw CChristine, Bartenfeld AThomas: Exercise evaluation guides for public health emergency preparedness. Homeland security affairs 2009, 5(3).

38. NIST/SEMATECH e-Handbook of Statistical Methods. , [http://www.itl.nist, gov/div898/handbook]. Accessed May 2011.

39. Ruter A, Vikstrom T: Improved staff procedure skills lead to improved management skills: An observational study in an educational setting. Prehosp Disaster Med 2009, 24(5):376-379.

40. Gryth D, Rådestad M, Nilsson $H$, et al: Evaluation of medical command and control using performance indicators in a full-scale, major aircraft accident exercise. Prehosp Disaster Med 2010, 25(2):118-124.

41. Kaji AH, Langford $V$, Lewis RJ: Assessing hospital disaster preparedness: a comparison of an on-site survey, directly observed drill performance, and video analysis of teamwork. Ann Emerg Med 2008, 52(3):195-201.

42. Kaji AH, Lewis RJ: Hospital disaster preparedness in Los Angeles County. Acad Emerg Med 2006, 13(11):1198-203.

43. Adini B, Goldberg A, Laor D, et al: Factors that may influence the preparation of standards of procedures for dealing with mass-casualty incidents. Prehosp Disaster Med 2007, 22(3):175-80.
44. Hsu EB, Jenckes MW, Catlett CL, et al: Effectiveness of hospital staff masscasualty incident training methods: A systematic literature review. Prehosp Disast Med 2004, 19(3):191-199.

45. Collander B, Green B, Millo Y, et al: Development of an "all-hazards" hospital disaster preparedness training course utilizing multi-modality teaching. Prehospital Disast Med 2008, 23(1):63-67.

46. Lynn M, Gurr D, Memon A, et al: Management of conventional mass casualty incidents: Ten Commandments for hospital planning. J Burn Care Res 2006, 27(5):649-58

47. Takahashi A, Ishii N, Kawashima T, et al: Assessment of medical response capacity in the time of disaster: the estimated formula of Hospital Treatment Capacity (HTC), the maximum receivable number of patients in hospital. Kobe J Med Sci 2007, 53(5):189-98.

48. Paul Aliyas Jomon, Hariharan Govind: Hospital capacity planning for efficient disaster mitigation during a bioterrorist attack. Proceedings of the 2007 Winter Simulation Conference, [http://portal.acm.org/citation.cfm? $i d=1351741]$. Accessed May 2011.

doi:10.1186/1757-7241-20-14

Cite this article as: Djalali et al:: Hospital incident command system (HICS) performance in Iran; decision making during disasters.

Scandinavian Journal of Trauma, Resuscitation and Emergency Medicine 2012 20:14.

\section{Submit your next manuscript to BioMed Central and take full advantage of:}

- Convenient online submission

- Thorough peer review

- No space constraints or color figure charges

- Immediate publication on acceptance

- Inclusion in PubMed, CAS, Scopus and Google Scholar

- Research which is freely available for redistribution

Submit your manuscript at www.biomedcentral.com/submit
C) Biomed Central 